\title{
Timing of Introduction of Complementary Foods — United States, 2016-2018
}

\author{
Katelyn V. Chiang, $\mathrm{MPH}^{1,2}$; Heather C. Hamner, $\mathrm{PhD}^{1}$; Ruowei Li, $\mathrm{MD}^{1}$; Cria G. Perrine, $\mathrm{PhD}^{1}$
}

The American Academy of Pediatrics (AAP) recommends introducing complementary foods (i.e., any solid or liquid other than breast milk or infant formula) to infants at approximately age 6 months (1). Although a consensus on ideal timing is lacking, most experts agree that introduction of complementary foods before age 4 months is too early because of infant gastrointestinal and motor immaturity $(1,2)$. In addition, early introduction prevents exclusively breastfed infants from reaching the recommended 6 months of exclusive breastfeeding (1) and might be associated with increased risk for overweight and obesity (3). Nationally representative data on complementary feeding are limited; state-level estimates have been previously unavailable. CDC analyzed 2016-2018 data from the National Survey of Children's Health (NSCH) $(\mathrm{N}=23,927)$ to describe timing of complementary feeding introduction and prevalence of early introduction of complementary foods before age 4 months (early introduction) among children aged $1-5$ years. Prevalence of early introduction was $31.9 \%$ nationally and varied geographically and across sociodemographic and infant feeding characteristics. These estimates suggest that many infants are introduced to complementary foods before they are developmentally ready. Efforts by health care providers and others who might influence infant feeding practices could help decrease the number of infants who are introduced to complementary foods too early.

$\mathrm{NSCH}$ is funded and directed by the Maternal and Child Health Bureau of the Health Resources and Services Administration. It is an annual web- and paper-based survey that collects information from parents and caregivers on their children's physical and emotional health, including infant nutrition, and is representative of noninstitutionalized U.S. children aged 0-17 years. During 2016-2018, the overall weighted response rate ranged from $37.4 \%$ to $43.1 \%$. Missing data for race/ethnicity (1.3\%) and household income relative to the federal poverty level (FPL) (16.3\%) were imputed using hot-deck and sequential regression imputation methods, respectively (4).

Timing of introduction of complementary foods was assessed by asking respondents with children aged 0-5 years "How old was this child when he or she was first fed anything other than breast milk or formula" (4). To ensure that children had sufficient time to have been introduced to complementary foods, analysis was restricted to children aged 1-5 years. Participants with reported introduction to complementary foods at age $\geq 12$ months (887) and those with other implausible feeding patterns (recalled breastfeeding duration, infant formula introduction, and complementary feeding introduction indicated $\geq 2$ months with no source of nutrition; 101) were excluded from analyses. The percentage of children who were introduced to complementary foods before age 4 months (early introduction) was calculated overall, at the state and regional levels, and by sociodemographic and infant feeding characteristics using SAS-callable SUDAAN (version 11.0; RTI International). Two-sample t-tests were used to identify statistically significant $(\mathrm{p}<0.05)$ differences across subgroups.

Among 23,927 children aged 1-5 years, the mean age at introduction of complementary foods was 4.7 months, with $31.9 \%$ of children introduced at $<4$ months, $51.0 \%$ at 4-6 months, and $17.1 \%$ at 7-11 months (Figure 1). Prevalence of early introduction varied across sociodemographic groups. Prevalence of early introduction was significantly higher among non-Hispanic Black (Black) children (40.5\%), compared with all other racial/ethnic groups and significantly lower among non-Hispanic Asian children (23.8\%), compared with all other groups except Hispanic children (29.9\%). Prevalence of early introduction was significantly lower among children living in households at $\geq 400 \%$ of the FPL $(28.5 \%)$ and whose mothers had a bachelor's degree or higher $(27.7 \%)$, compared with all other household FPL and maternal education groups. Early introduction also differed significantly by infant milk feeding status at age 4 months: prevalence of early introduction was $18.5 \%$ among children receiving only breast milk for milk feeds, $32.1 \%$ among those receiving breast milk and infant formula, and $41.6 \%$ among those receiving only infant formula for milk feeds (Table). At the state level, prevalence of early introduction ranged from $18.0 \%$ in New Mexico to $49.0 \%$ in Mississippi. In 34 states, $\geq 30 \%$ of children were introduced to complementary foods before age 4 months, including 14 states in which prevalence of early introduction was at least 35\% (Supplementary Table, https://stacks.cdc.gov/view/ cdc/95035) (Figure 2).

\section{Discussion}

Nearly one in three $(31.9 \%)$ U.S. infants is introduced to complementary foods before age 4 months, with a higher prevalence of early introduction among Black infants and infants of mothers and households at lower socioeconomic status. Reasons for early introduction to complementary foods are not fully understood; however, many early introducing mothers have reported believing that their infant was old enough to begin consuming solids (5). This suggests a perception of 


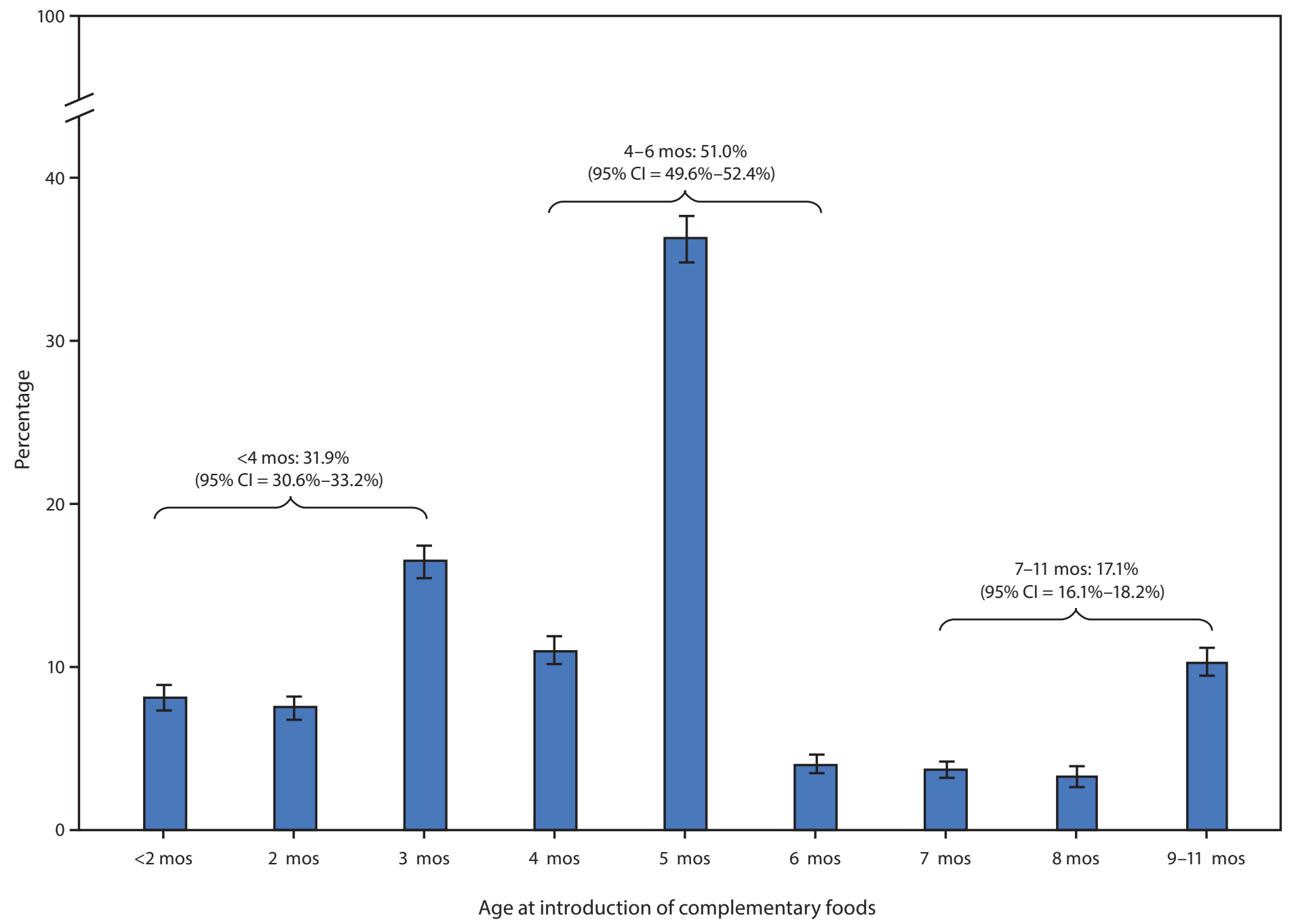

Abbreviation: $\mathrm{Cl}=$ confidence interval.

* $95 \% \mathrm{Cls}$ are indicated by error bars.

infant readiness for complementary feeding before the infant is actually ready and a potential lack of awareness of feeding recommendations, health effects associated with early introduction, and signs of developmental readiness. In general, infants show outward signs of readiness for complementary feeding when they can sit up on their own with good head control, show interest in mealtimes, are hungry in between feedings, and no longer have "tongue-thrust" or extrusion reflex, usually at approximately age 4-6 months (2).

Not only do younger infants lack the physiologic development to safely consume complementary foods, infants who are introduced to complementary foods too early have increased risk for multiple associated health conditions (1). Early introduction to complementary foods prevents infants from meeting the recommended 6 months of exclusive breastfeeding, decreasing the benefits both mothers and infants derive from exclusive breastfeeding. Compared with exclusive breastfeeding for 6 months, exclusive breastfeeding for 3-4 months followed by mixed breastfeeding and complementary feeding is associated with increased risk for gastrointestinal infection and slower maternal weight loss after birth (G). Further, limited evidence also suggests introduction to complementary foods before age 4 months might increase later overweight and obesity risk (3).

Health care providers can help increase awareness of recommended timing of introduction of complementary foods by employing consistent messaging in accordance with AAP recommendations and stressing the importance of developmental readiness when discussing complementary feeding with families (1). Resources are available to help health care providers engage with and educate families to better navigate the 
TABLE. Percentage of infants introduced to complementary foods before age 4 months, by sociodemographic characteristics, infant milk feeding status at age 4 months, and region among children aged 1-5 years - National Survey of Children's Health, United States, 2016-2018

\begin{tabular}{|c|c|c|c|}
\hline Characteristic & Total no.* & $\%$ Introduced early ${ }^{\dagger}$ & $95 \% \mathrm{Cl}^{\dagger}$ \\
\hline Total & 23,927 & 31.9 & $(30.6-33.2)$ \\
\hline \multicolumn{4}{|l|}{ Race/Ethnicity $\$$} \\
\hline Hispanic & 2,626 & 29.9 & $(26.3-33.7)$ \\
\hline White, non-Hispanic & 16,853 & 31.5 & $(30.2-32.9)$ \\
\hline Black, non-Hispanic & 1,211 & 40.5 & $(35.7-45.4)$ \\
\hline Asian, non-Hispanic & 1,125 & 23.8 & $(19.1-29.1)$ \\
\hline Other/Multiracial, non-Hispanic & 2,112 & 33.2 & $(29.5-37.1)$ \\
\hline \multicolumn{4}{|l|}{ Maternal age group (yrs) $)^{\mathfrak{n}, * *}$} \\
\hline $18-29$ & 4,634 & 34.0 & $(31.1-37.1)$ \\
\hline $30-39$ & 14,201 & 28.8 & $(27.3-30.5)$ \\
\hline$\geq 40$ & 3,412 & 33.3 & $(30.0-36.8)$ \\
\hline \multicolumn{4}{|l|}{ Maternal highest education level $\left.\right|^{\uparrow,+十}$} \\
\hline High school diploma or less & 2,544 & 34.3 & $(30.6-38.1)$ \\
\hline Some college & 5,962 & 33.4 & $(30.8-36.1)$ \\
\hline Bachelor's degree or more & 13,664 & 27.7 & $(26.3-29.1)$ \\
\hline \multicolumn{4}{|l|}{ Household income §§ $^{\S}$} \\
\hline$<100 \% \mathrm{FPL}$ & 2,429 & 35.2 & $(31.0-39.6)$ \\
\hline $100 \%-199 \% \mathrm{FPL}$ & 3,745 & 34.3 & $(30.9-37.8)$ \\
\hline $200 \%-399 \% \mathrm{FPL}$ & 7,662 & 31.7 & $(29.4-34.0)$ \\
\hline$\geq 400 \% \mathrm{FPL}$ & 10,091 & 28.5 & $(26.7-30.4)$ \\
\hline \multicolumn{4}{|l|}{ Infant milk feeding status at age 4 mos } \\
\hline Breast milk feeding only & 9,085 & 18.5 & $(16.9-20.2)$ \\
\hline Infant formula feeding only & 9,567 & 41.6 & $(39.5-43.8)$ \\
\hline Mixed breast milk and infant formula feeding & 4,863 & 32.1 & $(29.1-35.2)$ \\
\hline \multicolumn{4}{|l|}{ Region $* * *,+t+$} \\
\hline Northeast & 4,093 & 33.8 & $(30.8-36.8)$ \\
\hline Midwest & 6,063 & 32.3 & $(30.2-34.4)$ \\
\hline South & 7,675 & 34.8 & $(32.6-37.0)$ \\
\hline West & 6,096 & 25.7 & $(23.0-28.7)$ \\
\hline
\end{tabular}

Abbreviations: $\mathrm{Cl}=$ confidence interval, $\mathrm{FPL}=$ federal poverty level.

* Denominators might not sum to total because of missing maternal sociodemographic or infant milk feeding status data.

† Percentages are weighted to account for complex survey design.

$\S$ The percentage of infants introduced to complementary foods before age 4 months among Hispanic children is significantly different from that of non-Hispanic Black children. The percentage introduced early among non-Hispanic White children is significantly different from that of non-Hispanic Black and non-Hispanic Asian children. The percentage introduced early among non-Hispanic Black children is significantly different from that of all other racial/ethnic groups. The percent introduced early among non-Hispanic Asian children is significantly different from that of non-Hispanic White, non-Hispanic Black, and non-Hispanic other/ multiracial children. The percentage introduced early among non-Hispanic other/multiracial children is significantly different from that of non-Hispanic Black and non-Hispanic Asian children.

" Maternal sociodemographic data might be missing because no mother was reported in the child's household or because information was not reported by respondent.

** The percentage of infants introduced to complementary foods before age 4 months among children of mothers aged 30-39 years is significantly different from that of children of mothers aged $18-29$ and $\geq 40$ years.

tt The percentage of infants introduced to complementary foods before 4 months among children of mothers with bachelor's degrees or higher is significantly different from that of children of mothers of all other highest education levels.

$\S \S$ The percentage of infants introduced to complementary foods before 4 months among children living at $\geq 400 \%$ FPL is significantly different from that of children living at all other household income levels.

१ๆ The percentage of infants introduced to complementary foods before age 4 months among children receiving only breast milk for milk feeds at age 4 months is significantly different from that of children receiving all other types of nutrition for milk feeds at age 4 months. The percentage introduced early among children receiving only infant formula for milk feeds at age 4 months is significantly different from that of children receiving all other types of nutrition for milk feeds at age 4 months. The percentage introduced early among children receiving both breast milk and infant formula for milk feeds at age 4 months is significantly different from that of children receiving all other types of nutrition for milk feeds at age 4 months.

*** U.S. Census Bureau classifications for regions.

${ }^{+t+}$ The percentage of children introduced to complementary foods before age 4 months among children living in the West is significantly different from that of children living in all other regions.

transition from milk feeds to family foods (7). Further, given the high prevalence of early introduction of complementary foods among infants receiving formula, targeted education to parents and caregivers of those receiving infant formula might be particularly helpful. Similar efforts by others who could influence infant feeding practices such as peer educators, early care and education staff members, and Special Supplemental Nutrition Program for Women, Infants, and Children (WIC) staff members might also help reduce early introduction.

Another nationally representative study of U.S. children, the 2009-2014 National Health and Nutrition Examination Surveys (NHANES), found a lower prevalence of early 
FIGURE 2. Percentage of children introduced to complementary foods before age 4 months among children aged 1-5 years National Survey of Children's Health, United States, 2016-2018

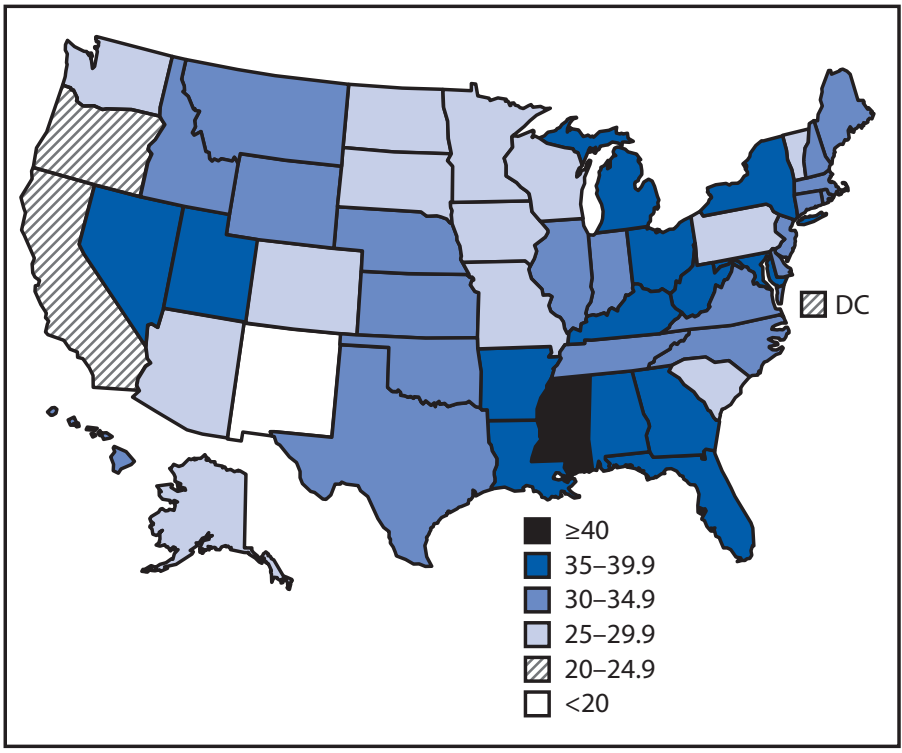

Abbreviation: $\mathrm{DC}=$ District of Columbia.

introduction $(16.3 \%)$ than the estimate from NSCH participants $(31.9 \%)$; however, similar patterns in early introduction by sociodemographic and infant feeding status characteristics were seen across both studies (8). The questions used to identify timing of complementary feeding introduction were the same for both studies. Some of the discrepancy might be explained by inherent differences in the surveys including representativeness of participants and response rates or by the different age ranges of studied children (6-36 months in NHANES compared with $1-5$ years in NSCH). Differences might also reflect changes over time in parental attitudes toward complementary feeding or health care provider advice because participants included in the NHANES and NSCH analyses were born during 2006-2014 and 2010-2018, respectively. Over the past decade, there has been growing awareness of the benefits of not delaying introduction of allergenic foods among children at high risk for food allergies to prevent the development of food allergies (9). It is possible that research might have been misinterpreted by parents, caregivers, and health care providers, leading to increases in early introduction to complementary foods in recent years. Further education on correct timing of introduction of allergenic foods and identification of children at high risk for food allergies might be needed to improve adherence to feeding recommendations.

The findings in this report are subject to at least four limitations. First, data might be affected by information bias. Though maternal recall of breastfeeding has been shown to have high validity and reliability, recall of solid and other liquid feeding

\section{Summary}

What is already known about this topic?

The American Academy of Pediatrics recommends introducing complementary foods at approximately age 6 months. Introduction before age 4 months is too early because infants are not developmentally ready for complementary foods. Early introduction prevents infants from reaching the recommended 6 months of exclusive breastfeeding.

What is added by this report?

Nearly one in three infants is introduced to complementary foods before age 4 months; prevalence of early introduction varies geographically and across sociodemographic and infant feeding characteristics.

What are the implications for public health practice?

Increasing awareness of and adherence to feeding recommendations could help reduce early introduction. Health care providers and others who might influence infant feeding practices should educate families on recommended timing of introduction of complementary foods.

might not be as reliable (10). However, participants with implausible feeding patterns were removed from the sample to account for potential misreporting of infant feeding information. Second, although multiply imputed, household FPL data might be misclassified. Third, data do not allow for analysis of types, amounts, or frequency of complementary foods offered; these are important markers of early child nutrition. Finally, small sample sizes limited the ability to conduct further sociodemographic analyses at the state level.

Introduction of complementary foods at the recommended time could help improve infant health and might play a role in prevention of overweight and obesity; however, nearly one third of infants are introduced to complementary foods too early. Early introduction also varies geographically and across sociodemographic and infant feeding characteristics, placing some infants, such as Black infants and infants of mothers and households of lower socioeconomic status, at increased risk for potential poor health outcomes related to early introduction of complementary foods. Increased education on complementary feeding recommendations, including the possible effects of early introduction and signs of developmental readiness, might help decrease the number of infants who are introduced to complementary foods too early.

Corresponding author: Katelyn V. Chiang, kchiang@cdc.gov, 404-498-0612.

\footnotetext{
${ }^{1}$ Division of Nutrition, Physical Activity, and Obesity, National Center for Chronic Disease Prevention and Health Promotion, CDC; ${ }^{2} \mathrm{Oak}$ Ridge Institute for Science and Education, Oak Ridge, Tennessee.
}

All authors have completed and submitted the International Committee of Medical Journal Editors form for disclosure of potential conflicts of interest. No potential conflicts of interest were disclosed. 


\section{References}

1. American Academy of Pediatrics Committee on Nutrition. Feeding the infant. In: Kleinman RE, Greer FR, eds. Pediatric nutrition. 8th ed. Itasca, IL: American Academy of Pediatrics; 2019.

2. Pérez-Escamilla R, Segura-Pérez S, Lott M. Feeding guidelines for infants and young toddlers: a responsive parenting approach. Durham, NC: Healthy Eating Research; 2017.

3. English LK, Obbagy JE, Wong YP, et al. Timing of introduction of complementary foods and beverages and growth, size, and body composition: a systematic review. AM J Clin Nutr, 2019; 109(Suppl_7):935S-55S.

4. Child and Adolescent Health Measurement Initiative. About the National Survey of Children's Health. Baltimore, MD: Data Resource Center for Child and Adolescent Health; 2019. https://www.childhealthdata.org/ learn-about-the-nsch/NSCH

5. Clayton HB, Li R, Perrine CG, Scanlon KS. Prevalence and reasons for introducing infants early to solid foods: variations by milk feeding type. Pediatrics 2013;131:e1108-14. PMID:23530169 https://doi. org/10.1542/peds.2012-2265
6. Kramer MS, Kakuma R. Optimal duration of exclusive breastfeeding. Cochrane Database Syst Rev 2012;8:CD003517. PMID:22895934

7. American Academy of Pediatrics Institute for Healthy Childhood Weight. Educating and engaging parents and families. Building a Foundation for Healthy Active Living. Itasca, IL: American Academy of Pediatrics Institute for Healthy Childhood Weight; 2020. https://ihcw.aap.org/ Pages/EFHALF_parents.aspx

8. Barrera CM, Hamner HC, Perrine CG, Scanlon KS. Timing of introduction of complementary foods to US infants, National Health and Nutrition Examination Survey 2009-2014. J Acad Nutr Diet 2018;118:464-70. PMID:29307590 https://doi.org/10.1016/j. jand.2017.10.020

9. Togias A, Cooper SF, Acebal ML, et al. Addendum guidelines for the prevention of peanut allergy in the United States: report of the National Institute of Allergy and Infectious Diseases-sponsored expert panel. Ann Allergy Asthma Immunol 2017;118:166-173.e7. PMID:28065802 https://doi.org/10.1016/j.anai.2016.10.004

10. Li R, Scanlon KS, Serdula MK. The validity and reliability of maternal recall of breastfeeding practice. Nutr Rev 2005;63:103-10. PMID:15869124 https://doi.org/10.1111/j.1753-4887.2005.tb00128 